Acta chir belg, 2005, 105, 560-566

\title{
Management of High Blood Pressure in Peripheral Arterial Disease
}

\author{
J.-M. Krzesinski \\ Department of Medicine, Nephrology Unit, University of Liège, Liège, Belgium.
}

Key words. Peripheral arterial disease (PAD) ; hypertension (HTA); blood pressure (BP) ; CAD (coronary arterial disease).

\begin{abstract}
Arterial hypertension (HTA) is a promoter of peripheral arterial disease (PAD) in association with other atherosclerotic risk factors. Systolic HTA is the most frequently noted form in such disease, secondary to marked increase in large artery stiffness.

The existence of PAD confers on the hypertensive patient a very high cardiovascular (CV) risk, requiring an intensive global therapeutical approach. Treating HTA is one of such beneficial actions. The optimal blood pressure (BP) to be reached is at least $<140 / 90 \mathrm{mmHg}$ but lower BP should be targeted if possible $(\leq 130 / 80 \mathrm{mmHg})$. First of all, a modification of the lifestyle and diet should be proposed. But to reach such a low target, different antihypertensive agents must be very often used in association. The inhibitors of the renin-angiotensin system constitute one of the main axis of such drug treatment, after having avoided the pitfall of renal artery stenosis.
\end{abstract}

\section{Introduction}

This overview will consider the relationship between hypertension and peripheral arterial disease (PAD). The following issues will be discussed : how is hypertension a promoter of PAD, what are the characteristics of hypertension in such patients, what is the benefit after treating hypertension, what is the optimal blood pressure target and what is the strategy to reach such goal ?

\section{Epidemiology of the relationship between hyperten- sion and peripheral arterial disease (PAD)}

Atherosclerosis is a systemic vascular disease. It is thus not surprising that there is a frequent coexistence in the adult population of CAD (coronary arterial disease), PAD and cerebral vascular accident (CVA). By classical definition, PAD is a condition in which atherosclerotic lesions of the lower limbs provoke symptoms of claudication, ischaemic pain or trophic lesions in the legs. Aronow et al. (1) observed in such patients older than 62 years that CAD was present in $21 \%$, CVA in $9 \%$ and PAD in $8 \%$ but $5 \%$ of this population presented simultaneously the 3 complications with an associated huge increased cardiovascular mortality.

The presence of PAD with intermittent claudication is commonly associated with CV risk factors. Among the modifiable ones, diabetes confers a risk for developing PAD X 2. For smoking, the odds ratio is X 2,5. For HTA and hypercholesterolaemia it is X 1.5 (Table 1) (2) Moreover, these factors are frequently aggregated in the same patient, increasing so the cardiovascular risk in a
Table 1

Odds ratio for developing PAD of modifiable and non modifiable risk factors

\begin{tabular}{|lc|}
\hline Modifiables factors & $\times 2.5$ \\
Smoking & $\times 2$ \\
Diabetes & $\times 1.5$ \\
Hypertension & $\times 1.5$ \\
Hypercholesterolaemia & \\
Non modifiable factors & $\times 2.5$ \\
Male sex & $\times 2$ \\
Age (per 10 y) & \\
\hline
\end{tabular}

Adapted from the Prevention of Atherothrombotic Disease Network, Arch Intern Med, 2003, 163 : 884-92.

very large proportion. From the Framingham study, KANNEL (3) observed (either in men or women) that $50 \%$ of the hypertensive patients presented 1 or 2 added risk factors, $20 \% 3$ risk factors and only less than $20 \%$ of the studied US population had isolated high blood pressure. This observation is rather the same in all the industrialized populations. Murabito et al. (4) studying the Framingham offspring population (1554 males and 1759 females with mean age of 59 years examined between 1995 and 1998), noted that odds ratio for significant associations between PAD (identified by ankle-brachial pressure index < 0.9) and HTA from multivariate analysis was 2.2 .

Atherosclerosis is first the result of endothelial dysfunction secondary to oxidative stress. Such a stress is due to several factors injuring the endothelial wall. High 
BP leads to increased shear stress creating endothelial damage and dysfunction.

For KANNEL (5), hypertensive male patients presented a PAD 2 times more frequently and this disease was even noted 3 times more frequently in women as compared to normotensives. Moreover, PAD was associated with considerable morbidity and mortality from $\mathrm{CV}$ causes, with an annual mortality of $25 \%$ when critical leg ischaemia (5).

\section{Importance of the ankle-brachial pressure index (ABP)}

When screening individuals for their cardiovascular risk, determination of the ankle-brachial pressure index (ABPI) has been proposed as a sensitive measure to detect PAD, the existence of which is characterized by a low value of this ABPI. Such situation has also been associated with increased CV morbidity and mortality (7).

In the most recent years, an important epidemiological aspect in CV risk was considered due to the epidemic obesity frequently associated to the metabolic syndrome. The definition of the later has been reviewed by several associations such as the NCEP ATP III (National Cholesterol Education Program-ATP III) (8-9) or the European Group for the study of Insulin Resistance (EGIR) (10).

For the American guidelines, such diagnosis is established when at least 3 of the following risk criteria are met : blood glucose $\geq 110 \mathrm{mg} / \mathrm{dl}(6.1 \mathrm{mmol} / \mathrm{l})$, waist circumference $\geq 102 \mathrm{~cm}$ in men, $\geq 88 \mathrm{~cm}$ in women, HDL cholesterol lower than $40 \mathrm{mg} / \mathrm{dl}(1 \mathrm{mmol} / \mathrm{l})$ in men and less than $50 \mathrm{mg} / \mathrm{dl}(1.3 \mathrm{mmol} / \mathrm{l})$ in women, triglycerides $\geq 150 \mathrm{mg} / \mathrm{dl}(1.7 \mathrm{mmol} / \mathrm{l})$ and a $\mathrm{BP}$ higher than $130 / 85 \mathrm{mmHg}$.

For the European guidelines (11), these criteria have been a bit different : a waist circumference $\geq 94 \mathrm{~cm}$ in men, $\geq 80 \mathrm{~cm}$ in women, a BP > 140/90 $\mathrm{mmHg}$ in both sex, a blood glucose $>110 \mathrm{mg} / \mathrm{dl}$, triglycerides $>150 \mathrm{mg} / \mathrm{dl}$ and a HDL cholesterol < $40 \mathrm{mg} / \mathrm{dl}$ in men as in women.

Considering this problem of metabolic syndrome, Olijhoek et al. (12) studied in the same time these metabolic syndrome components and potential existence of a low ABPI (Table 2). These authors observed that only $2 \%$ of the population presented a low ABPI $(\leq 0.9)$ in at least one leg when no metabolic syndrome component was present. This percentage, however, increased to $12 \%$ when 3 components were noted and to $22 \%$ when all the 5 components were present. The prevalence of microalbuminuria increased also in parallel with the number of metabolic syndrome components. In this order of idea, the components of the metabolic syndrome are frequently noted in chronic kidney disease
Table 2

Components of the metabolic syndrome in relation to decreased ABPI

\begin{tabular}{|c|c|}
\hline $\begin{array}{c}\text { Metabolic syndrome } \\
\text { components (n) }\end{array}$ & $\begin{array}{c}\text { Decreased ABPI* } \\
(\%)\end{array}$ \\
\hline 0 & 2 \\
1 & 10 \\
2 & 12 \\
3 & 11 \\
4 & 18 \\
5 & 22 \\
\hline
\end{tabular}

Decreased ABPI : Ankle Brachial Pressure Index $\leq 0.90$ in at least one leg (age- and sex adjusted).

* Patients with clinical symptomatic PAD were excluded from analyses.

Adapted from Oцinнeк J. et al. Eur Heart J, 2004.

(CKD) (13) and CKD could also be caused by such syndrome. For its part, CKD is frequently associated to PAD (14).

Recently, it was observed that subclinical PAD, detected by a ABPI less than or equal to 0.9, was common $(32 \%)$ in non dialysed patients with CKD, related to age, male sex and a more severe degree of renal insufficiency (15). In this study, however only $30 \%$ yet presented a clinical picture compatible with intermittent claudication.

\section{Characteristics of the hypertension in PAD patients}

A strong association has been more often demonstrated between elevated systolic BP (than the diastolic component) and intermittent claudication.

GAGNON et al. (16) studied more than 4000 men aged 35-64 years who did not suffer from PAD at the beginning period of a follow-up of 16 years. For those individuals in the highest quintile of SBP (> $152 \mathrm{mmHg}$ ), the RR was found to be 2.7 to develop intermittent claudication, but for those in the highest quintile for DBP (> $92 \mathrm{mmHg}$ ), the RR was only 1.5 .

In the same order of idea, VoGT et al. (17) evaluated 575 patients with unisegmental PAD, 587 with multi segmental disease and 243 patients without apparent disease. Elevated SBP was found to be a key risk factor for arterial disease at a proximal level : isolated aortic, iliac and femoropopliteal arterial disease, in both men and women. For distal arterial disease, in women, elevated SBP was also the major correlate, while in men, the main correlate was especially an history of diabetes mellitus.

The explanation for increasing more the systolic component of $\mathrm{BP}$ is the following :

In PAD, when the central aortic pressure waveforms are recorded, derived from percutaneous tonometry tracings, as conduit arteries are stiffening, the second 
systolic peak becomes more prominent due to increase in pulse wave velocity (PWV) and earlier pulse wave reflection. Therefore, the energy of the reflected wave will combine at an earlier time with the antegrade pulse wave, thus increasing the augmentation index. This parameter is influenced by the reflective properties of the arterial system and is strongly dependent on PWV and the travelling distance of pressure waves (body height).

The SBP increases as a result of this abnormality and the DBP decreases at the same time. Thus, pulse pressure is generally high in PAD and such abnormality is of importance when CV risk is considered. Indeed, SAFAR et al. (18) analysing data from 3 important studies in systolic HTA treating old patients (EWPHE, SYSTEUR and SYST CHINA) observed that the CV risk increased with the SBP level but for each level considered, the risk was higher when the pulse pressure was higher.

In the SHEP trial (systolic HTA in the elderly program), the prevalence of lower extremity arterial disease was assessed in a population of hypertensive patients older than 60 years and with SBP > $160 \mathrm{mmHg}$ (19).

Using the criteria of clinical symptoms of intermittent claudication, a prevalence of $6.5 \%$ was observed, but using the criterion of an ABPI $\leq 0.9$, this prevalence reached $26.7 \%$ linked to age, smoking and low HDL cholesterol.

In this SHEP study, and for a mean follow-up of 16 months, Newman et al. (20) noted that mortality and morbidity were higher in 1537 patients with systolic HTA and low ABPI as compared to patients with ABPI higher than 0.9 : for total deaths, the RR adjusted for age and sex was 3.8 , for the CHD deaths this risk was increased by 3.2 and for CVD deaths, the RR was increased by 3.7 in the low ABPI group.

\section{Is treating HTA beneficial in such PAD patients ?}

The answer is of course positive. Treating HTA in high $\mathrm{CV}$ risk is very useful and profitable from a pharmacoeconomic point of view. But, the beneficial effects of treating HTA on atherosclerosis disease or on CV events have not been directly evaluated in patients presenting both PAD and HTA and this is also the case for PAD and diabetes. However, observational data and those derived from trials in people with other manifestations of $\mathrm{CV}$ disease may be probably generalized to support the importance of treating key risk factors such as HTA in PAD.

Indeed, the large intervention for treating HTA trials have not specifically targeted patients with PAD. In the absence of such data, consensus still supports the management of HTA in these patients as PAD is included in the group of markers of CV disease and calls for maintenance of BP level less than 130-80 in these patients (21).
According to the recent ESH-ESC Guidelines (11), in stage 1 HTA with additional CV disease risk factors, achieving a sustained $12 \mathrm{mmHg}$ reduction in SBP over 10 years will prevent 1 death for every 11 patients treated. However, in the detailing of the parameters defining target organ damage (TOD) and/or associated clinical complications linked to the BP risk evaluation, these new guidelines have included the presence of PAD either symptomatic or detected by the presence of plaques or even by a ABPI $\leq 0.9$. The existence of TOD or associated clinical conditions (ACC) with a BP measured in the range of the high normal BP group (130$139 / 85-89 \mathrm{mmHg}$ ) gives a high added or even a very high added risk to the patient and this position could be an argument to decide very quickly initiation of antihypertensive agent prescription.

In the JNC 7 even, СHOBANIAN et al. (22) considered PAD equivalent in risk to ischaemic heart disease.

\section{What is the optimal BP target and how to reach it?}

The goals for treatment of HTA in PAD patients are to reduce CVD and renal morbidity and mortality.

In patients suffering from diabetes or $\mathrm{CKD}$, the target BP should be $<130 / 80 \mathrm{mmHg}$.

In patients with a high $\mathrm{CV}$ risk such as the patients with PAD, this threshold could be proposed : however, a too large and rapid reduction of BP could worsen intermittent claudication and perhaps unmask a coronary heart disease, frequently associated. This can be the case whatever drug treatment used and no evidence exists that beta-blockers are particularly more culpable in this problem of aggravating leg symptoms.

In type 2 diabetes, MEHLER et al. (23) noted that intensive control BP reduced more the risk of $\mathrm{CV}$ events when PAD was present. They compared a moderate target BP under treatment (BP $<140 / 85 \mathrm{mmHg}$ ) and an intensive target $(\mathrm{BP}<130 / 80 \mathrm{mmHg})$ dividing their population according to the baseline ABPI. After adjusting for age, sex, duration of diabetes, smoking habits, total cholesterol level, history of myocardial infarction or stroke, and adjusted cornell voltage, patients randomized to moderate treatment had an inverse relationship between ABPI and events $(p=0.009)$. On the other hand, patients intensively treated (with nisoldipine or enalapril in this study) had no such relationship between ABPI and events, demonstrating the protective effects of intensive BP treatment in such high CV risk patients.

\section{What treatment should be proposed first ?}

1. The non-pharmacological approach has a central role in treating HTA in such patients presenting PAD. Measures affecting the lifestyle have indeed a role in BP reduction but also, for several approaches, an added 
Table 3

Relative effectiveness of non-pharmacological measures in hypertension with PAD

\begin{tabular}{|l|c|c|}
\hline & $\begin{array}{c}\text { Blood pressure } \\
\text { reduction }\end{array}$ & $\begin{array}{c}\text { Coronary } \\
\text { protection }\end{array}$ \\
\hline Weight control & +++ & ++ \\
Salt restriction & ++ & $?$ \\
Moderate exercise & ++ & ++ \\
Mediterranean diet & + & +++ \\
Dietary fish & \pm & +++ \\
Stopping smoking & - & +++ \\
\hline
\end{tabular}

useful coronary protection profile (Table 3). For the BP reduction, weight control and salt restriction are the most powerful, but for coronary protection (and diffuse arterial), mediterranean diet, dietary fish, omega 3 consumption and stopping smoking have the most important effect. However, moderate exercise and weight control associated with a moderate alcohol intake offer a protective and interesting effect on the coronary events. Concerning salt intake, GATES et al. (24) noted that sodi- um restriction rapidly improves large elastic arterial compliance in older adults with systolic HTA, this parameter being particularly decreased in PAD.

2. In JNC 7, any class of antihypertensive drugs could be used in most patients with PAD (22). Besides the important non-pharmacological approach, the algorithm for treatment of HTA, when PAD exists, insists on introducing antihypertensive drugs when the BP was not yet in the normal range (Fig. 1). The preferred initial drug choice could be one drug at its full dosage or a two drugs combination at a lower dosage. After several weeks, if the patient is not yet at the goal BP, an optimization of the dosages or adding a third drug has to be proposed until goal BP is achieved. However, BP should decrease slowly in these patients. In paralell, other risk factors must also be managed aggressively, such as by statine and aspirin prescriptions. In the UKPDS (United Kingdom Prospective Diabetes Study) performed in type 2 diabetes, if diabetes end-points and risks of stroke were significantly reduced by tight BP, risk for myocardial infarction or for amputation related to PAD were not significantly reduced (24).

First step

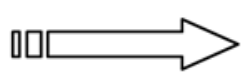

Lifestyle Modifcations

(see Table 3)

Not at Goal Blood Pressure $(\leq 130 / 80 \mathrm{mmHg})$
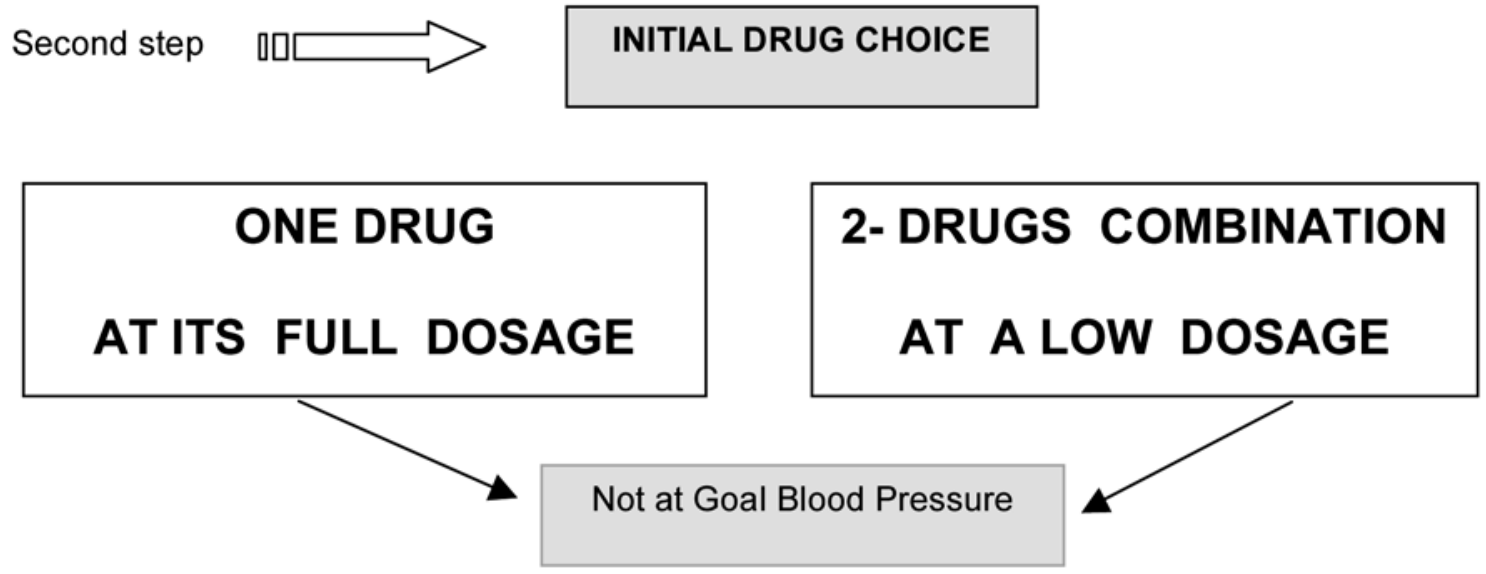

Third step

Fig. 1

Algorithm for Treatment of Hypertension when PAD coexists

Adapted from The sixth report of the Joint National Committee Arch Intern Med, 1997, 157 : 2413-45. 


\section{Is there an ideal antihypertensive drug with addi-} tional properties?

The different antihypertensive classes are possible candidates for treating high BP in PAD population.

Beta-blockers (BB) are known to reduce mean BP, cardiac output and blood flow in the peripheral circulation including exercising muscles, due to blockade of the vascular B2 receptors. Non-selective BB could thus worsen the symptoms of PAD. However, BOGAERT \& Clement (26) were unable to observe any influence of beta-blockers on walking distance in patients with chronic intermittent claudication, comparing propanolol, metoprolol and placebo.

Solomon et al. (27) found neither adverse nor beneficial effects with atenolol, a cardioselective $\beta 1 \mathrm{BB}$ as compared to the calcium channel antagonist nifedipine on PAD when given as a single therapy. However, the combination of both decreased walking ability by $9 \%$. But we must be cautious not to apply such a BB treatment to too severely affected PAD patients. Newer BB with vasodilating properties could yet be more beneficial. This theoretical advantage however deserves prospective studies.

Angiotensin converting enzyme inhibitors (ACEI) may constitute an excellent choice for antihypertensive therapy in patients with PAD, particularly when some collateral flows exist. HIRSCH \& DuPREZ (28) reviewed the interest of ACEI treatment in PAD and concluded that such therapy could improve clinical symptoms in PAD. For instance, ACEI can also improve arterial compliance of the large arteries leading to a decreased in the cardiac work. However, attention should also be paid to renal function in the case of coexistence of renal artery stenosis (RAS) which can frequently enough occur in such patients. In this context, it must be remembered that lower limb occlusion disease was noted in 20 to $40 \%$ of patients suffering from RAS $\geq 50 \%$ (29-30).

In the HOPE trial (Heart Outcomes Prevention Evaluation study) (31), from a total of 9297 patients, 4051 suffered from PAD. The high dosage of ramipril $(10 \mathrm{mg})$ was particularly useful in protecting such high $\mathrm{CV}$ risk patients with PAD. This treatment reduced $\mathrm{CV}$ morbidity and mortality by around $25 \%$ more than in the placebo group. Patients did not have to be hypertensive to be included in this study, and the observed risk reduction could not be accounted for by the relatively modest reduction of BP. The implication of the HOPE study is that most patients with PAD would benefit from an ACEI, provided that treatment is not associated with a deterioration of renal function due to occult RAS.

In the VALUE study (32), 14\% presented PAD and all of these patients had high CV risk. In this trial, the calcium channel blocker (CCB) amlodipine was, during the first months of the study, more beneficial for the $\mathrm{CV}$ protection than the angiotensin II receptor blocker (ARB), valsartan, probably due to a more rapid and prominent antihypertensive effect, and this was particularly noted for the problem of myocardial infarction complications.

As summarized in a recent review (28), ACEI were remembered to provide vascular protective effects such as improvement of endothelial dysfunction, BP lowering and promotion of angiogenesis in ischaemic lesion. At the clinical point of view, they increase the ABPI, the peripheral blood flow (demonstrated in small scale clinical investigations) and decrease morbidity and mortality (proven in large-scale prospective investigations such as HOPE or EUROPA for European trial On reduction of cardiac events with Perindopril in stable coronary Artery disease investigators) (33). In this later large study, ACEI perindopril at high dosage among patients with stable coronary heart disease without heart failure significantly improved outcomes. In this study, $7 \%$ of the population suffered from PAD.

In the LIFE study (Losartan Intervention For Endpoint reduction in hypertension study) (34), the ARB losartan was also found to significantly reduce the composite endpoint of $\mathrm{CV}$ death, myocardial infarction or stroke in patients with hypertension and LVH compared with atenolol. Although the results seem to be very comparable to the HOPE data, when analysing the prevalence of coronary or peripheral revascularization procedures, a pre-specified secondary endpoint, no difference was found between the 2 treatment groups. The total number of participants with a history of PAD included in the LIFE study was much lower than in the HOPE trial (6\% or $8.5 \%$ when considering only systolic HTA) (35). However, the use of losartan for the treatment of HTA in patients with PAD can be recommended but possible additional protective vascular effects of losartan and other ARB on PAD are still to be determined.

Thus, in theory and to summarize, all the 6 classes of drugs could be a first optimal choice, but ACEI and ARB could be prefered if no RAS is suspected. Calcium channel blocker could be another excellent option when RAS is suspected.

B1 selective $\mathrm{BB}$ or non selective $\mathrm{BB}$ with additive vasodilating properties could also be useful if a coronary problem but no critical leg ischaemia is present.

However, very often, association of several antihypertensive agents are necessary to reach the target systolic BP in such PAD patients with alteration of large arteries and systolic HTA.

Diuretics could also be used but at a low dosage. Indeed, ERKENs et al. (36) noted that the thiazide diuretic use was associated with excess amputations. Thus we must keep in mind this and be cautious when using high dosage of such diuretic treatment in PAD with HTA and diabetes mellitus. 


\section{Conclusion}

The definition of PAD is equivalent in risk to ischaemic heart disease as recently considered by the JNC 7 report (22). Systolic HTA is frequently noted in such patients with vascular alteration, due to arterial stiffness. To reduce the $\mathrm{CV}$ risk, BP must be treated when higher than $140 / 90 \mathrm{mmHg}$ first by a non-pharmacological approach. If the BP is not yet reduced to the target (which ideally could be less than $130 / 80 \mathrm{mmHg}$ ), a drug therapy must be started.

Any class of antihypertensive drugs can be used but a preferential option should be ACEI (or ARB). The CCB could be another option if RAS is suspected. However, an association of both drug classes is frequently required.

All the other CV risk factors should also be managed aggressively in parallel, as recently extrapolating the demonstrated results in high cardiovascular risk hypertensive patients included in the ASCOT trial (37).

\section{References}

1. Aronow W., Ahn C. Prevalence of coexistence of coronary artery disease, peripheral arterial disease, and atherothrombotic brain infarction in men and women $\geq 62$ years of age. Am J Cardiol, 1994, $74: 64-5$.

2. Critical issues in peripheral arterial disease detection and management : a call to action. Arch Intern Med, 2003, 163 : 884-92.

3. KANNEL W. B. Risk stratification in hypertension : new insights from the Framingham Study. Am J Hypertens, 2000, 13 : 3s-10s.

4. Murabito J., Evans J., Nieto K., Larson M., Levy D., Wilson P. Prevalence and clinical correlates of peripheral arterial disease in the Framingham Offspring Study. Am Heart J, 2002, 143 : 961-5.

5. KANNEL W. B. Potency of vascular risk factors as the basis for antihypertensive therapy. Eur Heart J, 1992, suppl G : 34-42.

6. Dormandy J. A., HeEck L., VIg S. The fate of patients with critical leg ischemia. Seminar Vasc Drug, 1999, 12 : 142-7.

7. Mohler E. R. Peripheral arterial disease : identification and implications. Arch Intern Med, 2003, 163 : 2306-14.

8. Expert panel on detection, evaluation and treatment of high blood cholesterol in adults. Executive summary of the third report of the National Cholesterol Education Program (NCEP) expert panel on detection, evaluation, and treatment of high blood cholesterol in adults (Adult Treatment Panel III). JAMA, 2001, 285 : 2486-97.

9. Third Report of the National Cholesterol Education Program (NCEP) Expert Panel on Detection, Evaluation, and treatment of High Blood Cholesterol in Adults (Adult Treatment Panel III) Final Report. Circulation, 2002, 106 : 3145-421.

10. European Group for the study of Insulin Resistance (EGIR) Diabetes Metab, 2002, 28 : 364-76.

11. Guidelines Committee. 2003 European Society of Hypertension European Society of Cardiology guidelines for the management of arterial hypertension. J Hypertens, 2003, 21 : 1011-53.

12. Olijhoek J., van Der GraAF Y., Banga J.-D. et al. The metabolic syndrome is associated with advanced vascular damage in patients with coronary heart disease, stroke, peripheral arterial disease or abdominal aortic aneurysm. Eur Heart J, 2004, 25 : 342-8.

13. Chen J., Muntner P., Hamm L. L. et al. Association of the metabolic syndrome and chronic kidney disease in U.S. adults. Ann Int Med, 2004, 140 : 167-74.

14. O'Hare A., Glidden D., Fox C., Hsu C. Y. High Prevalence of peripheral arterial disease in persons with renal insufficiency. Circulation, 2004, 109 : 320-3.
15. Garcia De Vinuesa S., Ortega M., Martinez P., Goicoechea M., Gomez CAMPdera F., LuÑo J. Subclinical peripheral arterial disease in patients with chronic kidney disease : prevalence and related risk factors. Kidney Int, 2005, 67 : S44-7.

16. Gagnon F., Dagenais G., Robitaille N. M., Lupien P. J. Impact of systolic and diastolic blood pressure on ischaemic vascular diseases in French-Canadian men from 1974 to 1990. Can J Cardiol, 1994, $10: 97-105$.

17. Vogt M. T., Wolfson S. K., Kuller L. M. Segmental arterial disease in the lower extremities. Correlates of disease and relationship to mortality. J Clin Epidemiol, 1993, 46 : 1267-76.

18. SAFAR M. Systolic blood pressure, pulse pressure and arterial stiffness as cardiovascular risk factors. Curr Opin Nephrol Hypertens, 2001, $10: 257-61$

19. Newman A. B., Stutton-Tyrrell K., Rutan G., Locher J., KULLER L. H. Lower extremities arterial disease in elderly subjects with systolic hypertension. J Clin Epidemiol, 1991, 44 : 15-20.

20. Newman A., Sutton-Tyrrell K., Vogt M., Kuller L. Morbidity and mortality in hypertensive adults with a low ankle/arm blood pressure index. JAMA, 1993, 270 : 487-9.

21. The sixth report of the Joint National Committee on prevention, detection, evaluation, and treatment of high blood pressure. Arch Intern Med, 1997, 157 : 2413-45.

22. Chobanian A., Bakris G., Black H. et al. The seventh report of the Joint National Committee on prevention, detection, evaluation, and treatment of high blood pressure. The JNC 7 report. JAMA, 2003, 289 : 2560-72

23. Mehler P., Coll J., Estacio R., Esler A., Schrier R., Hiatt W. Intensive blood pressure control reduces the risk of cardiovascular events in patients with peripheral arterial disease and type 2 diabetes. Circulation, 2003, 107 : 753-6.

24. Gates P. E., Tanaka H., Hiatt W. R., Seals D. R. Dietary sodium restriction rapidly improves large elastic artery compliance in older adults with systolic hypertension. Hypertension, 2004, 44 : $35-41$.

25. UK Prospective Diabetes Study Group. Tight blood pressure control and risk of macrovascular and microvascular complications in type 2 diabetes: UKPDS 38. BMJ, 1998, 317 : 703-13.

26. Bogaert M. G., Clement D. L. Lack of influence of propanolol and metoprolol on walking distance inpatients with chronic intermittent claudication. Eur Heart J, 1983, 4 : 203-4.

27. Solomon S. A., Ramsay L. E., Yeo W. W., Parnell L., MorisJoNES W. Beta-blockade and intermittent claudication : placebo controlled comparison of atenolol and nifedipine and their combinations. BMJ, 1991, $303: 1100-4$.

28. Hirsch A. T., Duprez D. The potential role of angiotensin-converting enzyme inhibition in peripheral arterial disease. Vasc Med, $2003,8: 273-8$

29. The Heart Outcomes Prevention Evaluation Study investigators. Effects of an angiotensin-converting-enzyme inhibitor, ramipril, on cardiovascular events in high-risk patients. $N$ Engl J Med, 2000, 342 : 145-53.

30. Shurrab A. E., MacDowall P., Wright J., Mamtora H., KALRA P. A. The importance of associated extra-renal vascular disease on the outcome of patients with atherosclerotic renovascular disease. Nephrol Clin Pract, 2003, 93 : c51-7.

31. Olin J. W., Melia M., Young J. R., Graor R. A., Risius B Prevalence of atherosclérotic renal artery stenosis in patients with atherosclerosis elsewhere. Am J Med, 1990, 88 : 46N-51N.

32. Julius S., KueldSen S., Weber M. et al. Outcomes in hypertensive patients at high cardiovascular risk treated with regimens based on valsartan or amlodipine : the VALUE randomised trial. Lancet, 2004, 363 : 2022-31.

33. Efficacy of perindopril in reduction of cardiovascular events among patients with stable coronary artery disease : randomised, double-blind, palceob-controlled, multicentre trial (the EUROPA study). Lancet, 2003, 362 : 782-8.

34. Dahlöf B., Devereux R. B., KJeldsen S. E. et al. Cardiovascular morbidity and mortality in the Losartan Intervention For Endpoint reduction in hypertension study (LIFE) study : a randomised trial against atenolol. Lancet, 2002, 359 : 995-1003.

35. KJELDSEN S., DAhlöF B., Devereux R. et al. Effects of losartan on cardiovascular morbidity and mortality in patients with isolated 
systolic hypertension and left ventricular hypertrophy. A Losartan Intervention For Endpoint reduction (LIFE) substudy. JAMA, 2002, 288 : 1491-8.

36. Erkens J., Klungel O., Stolk R., Spoelstra J., Grobbee D., LEUFKENS $\mathrm{H}$. Antihypertensive drug therapy and the risk of lower extremity amputations in pharmacologically treated type 2 diabetes patients. Pharmacoepidemiology Drug Safety, 2004, 13 : $139-46$.

37. Sever P., Dahlöf B., Poulter N. et al. Prevention of coronary and stroke events with atorvastatin in hypertensive patients who have average or lower-than-average cholesterol concentrations, in the
Anglo-Scandinavian Cardiac Outcomes Trial-Lipid Lowering Arm (ASCOT-LLA) : a multicentre randomised controlled trial. Lancet, 2003, 361 : 1149-58.

\section{J.-M. Krzesinski}

Department of Medicine, Nephrology Unit

C.H.U. Liège, University of Liège

4000 Liège, Belgium 